\title{
Pfadabhängigkeit, Konvergenz oder regulativer Wettbewerb: Determinanten der Außenwirtschaftsliberalisierung, 1978-2002*
}

\author{
Christian W. Martin / Gerald Schneider
}

\section{Einleitung}

Seit der Verschuldungskrise des letzten Jahrhunderts, spätestens aber mit dem Ende des Kalten Krieges zeichnet sich ein neuer Konsens ab, wie Regierungen die Außenwirtschaftspolitik ihres Landes gestalten sollen. Galt gerade unter Entwicklungsländern eine Strategie der Abschottung gegenüber der Weltwirtschaft bis zu diesem Zeitpunkt als probates Mittel der "nachholenden Entwicklung", so richten sich heute die Erwartungen auf eine Integration in die globale Ökonomie. Außenhandelsliberalisierung und Öffnung des Kapitalverkehrs sollen den Wohlstand mehren und entwicklungsschwache Länder zur Riege der OECD-Staaten aufschließen lassen, die sich teilweise schon vorher dem internationalen Wettbewerb geöffnet hatten.

Wir demonstrieren in unserem Beitrag, dass die nahe liegende These von einer einheitlichen „Konvergenz" der Außenwirtschaftspolitiken auf ein niedriges Regulierungsniveau empirisch nicht haltbar ist. Zwar weisen einzelne Gruppen von Staaten eine Abnahme der Varianz des Regulierungsgrades auf; für das Sample insgesamt lassen sich solche Prozesse jedoch nicht nachweisen. Zurückweisen können wir auch die Behauptung, dass gerade die autarken Staaten das Regulierungsniveau ihrer Konkurrenten durch eine Deregulierung zu unterbieten versucht hätten, so dass sich in der Summe die Öffnung der Außenwirtschaft als "Wettlauf nach unten" beschreiben ließe. ${ }^{1}$ Wir zeigen überdies theoretisch, dass die These eines Regulierungswettbewerbes auf sehr speziellen Annahmen zur innenpolitischen Autonomie der Regierungen beruht.

Die empirische Analyse demonstriert in Abgrenzung zu den Konvergenz- und Regulierungswettbewerbsthesen, dass die wirtschaftspolitische Globalisierung bis jetzt sehr uneinheitlich verlaufen ist und dass die Ausgangsbedingungen eines Staates weitgehend über dessen Öffnungserfolg entscheiden. Unsere Analyse bezieht sich auf ein weltweites

\footnotetext{
* Eine vorläufige Version dieses Papiers wurde auf der PVS-Autorenkonferenz „Transfer, Diffusion und Konvergenz von Politiken“, Universität Hamburg, 10./11. Februar 2006, präsentiert. Wir danken dem Publikum, unserem Diskutanten Achim Kemmerling, einem anonymen Gutachter sowie den Herausgebern für hilfreiche Kommentare. Vorarbeiten zu diesem Artikel wurden von der Volkswagenstiftung finanziert. Die in dieser Analyse verwendeten Daten sind auf der folgenden Webseite verfügbar: http://www.uni-konstanz.de/FuF/Verwiss/GSchneider/ downloads/daten.htm.

1 Wir verstehen hier den "Wettlauf nach unten" rein empirisch und untersuchen, ob Staaten die regulativen Hürden senken, die dem freien Austausch von Gütern und Kapital entgegenstehen. In diesem Sinne unterscheidet sich unsere Analyse auch nicht von den zahllosen Untersuchungen, die sich mit Steuerwettbewerb beschäftigen. Dass ein "Wettlauf nach unten" je nach dem unterschiedliche Wohlfahrtsimplikationen haben kann, ist in dieser positiven Analyse unerheblich. Wesentlich ist für uns hier nur, die Bedingungen zu klären, unter denen ein regulativer Wettbewerb unter Nationalstaaten auch bei marktschaffenden Maßnahmen möglich ist.
} 
Sample von Nationalstaaten und berücksichtigt das Niveau der Regulierung in der Außenwirtschaftspolitik an Stelle von Ergebnisvariablen wie dem Außenhandelsquotienten, die Grundlage der meisten Globalisierungsstudien sind.

Aus der statistischen Analyse lassen sich verschiedene Gesetzmäßigkeiten ableiten. Erstens übt die Restriktivität der Außenwirtschafsregulierung zum Untersuchungsbeginn einen durchweg positiven und signifikanten Einfluss auf die Regulierung zu späteren Zeitpunkten aus. Dieser Zusammenhang kann als Ausdruck pfadabhängiger Politikprozesse verstanden werden. Ursprünglich hoch regulierte Länder tendieren damit zu einer weiterhin restriktiven Regulierung. Dennoch senkt zweitens ein hohes Ausgangsregulierungsniveau das Ausmaß, in dem ein Staat nachfolgend seine Außenwirtschaftspolitik regelt. In dieser Betrachtungsweise stellt die Veränderung der Restriktivität das beobachtete Phänomen dar. Drittens beeinflussen die Rechte und Pflichten, die aus der Mitgliedschaft in internationalen Organisationen erwachsen, sowohl das Regulierungsniveau als auch seine Veränderung über die Zeit. Unser Blick gilt dabei besonders den IWF-Statuten, mit denen sich Mitglieder des IWF auf eine Öffnung des Kapitalverkehrs festlegen. Viertens erhöht die Restriktivität der Regulierung in anderen Staaten die Abschottung im beobachteten Staat. Dieser Effekt deutet auf strategisches Handeln hin. Zusätzlichen Halt findet diese Interpretation in der Tatsache, dass dieser Effekt über unterschiedliche Regionen hinweg unterschiedlich stark ausfällt und in teilweise konträre Richtungen verweist.

Aus den ersten zwei Beobachtungen lassen sich Antworten auf die übergeordnete Frage nach möglichen Konvergenzphänomenen gewinnen. Zum einen beruht unsere Interpretation darauf, dass die Varianz des Regulierungsgrades über die Zeit zugenommen hat. Zum anderen berücksichtigt die Erklärung, dass das hohe Ausgangsniveau der Regulierung zu einer stärkeren Senkung der Außenwirtschaftsregulierung führt. Doch diese Öffnung ist insofern pfadabhängig, als der deregulierende Effekt eines hohen regulativen Niveaus zu Beginn des Beobachtungszeitraumes nicht ausreicht, um den restringierenden Effekt des Ausgangsniveaus zu kompensieren. Mit anderen Worten: Stark regulierte Staaten verringern zwar die Restriktivität ihrer Außenwirtschaftspolitik in einem größeren Ausmaß als Staaten, die zu Beginn des Beobachtungszeitraums weniger restriktive Politiken verfolgten. Dieser Effekt fällt aber nicht groß genug aus, um eine Konvergenz im Sinne einer Reduzierung der Dispersion von Außenwirtschaftspolitiken herbeizuführen. In der Sprache der Wachstumstheorie ausgedrückt: Was wir beobachten, ist $\beta$-Konvergenz, aber keine $\sigma$-Konvergenz. Zugleich folgt daraus, dass Konvergenz auf ein niedriges Regulierungsniveau dort zu erwarten ist, wo das Niveau der Ausgangsregulierung hinreichend gering war. Diese Aussage deckt sich mit den Befunden für das Sub-Sample der OECD-Staaten, die einen eigentlichen Konvergenzclub auf dem Gebiet der Außenwirtschaftspolitik bilden.

Der Rest des Aufsatzes gliedert sich wie folgt: Im nächsten Abschnitt entwickeln wir ein einfaches Modell, das uns die drei unterschiedlichen Globalisierungsstrategien verstehen lässt. Wir leiten aus diesem theoretischen Rahmen Hypothesen ab, die wir in dem darauf folgenden Abschnitt einer empirischen Überprüfung unterziehen. Ein abschließender Teil resümiert. 


\section{Konvergenz und Divergenz von Außenwirtschaftspolitik}

Die Globalisierungsdiskussion hat bis jetzt weitgehend argumentiert, dass die weltwirtschaftliche Integration zu zwei unterschiedlichen Phänomenen führen kann: Konvergenz oder Divergenz nationalstaatlicher Politiken. Von Konvergenz sprechen wir im Kontext der Außenwirtschaftspolitik dann, wenn die Varianz der außenwirtschaftlichen Regulierung zwischen den Staaten über die Zeit abnimmt. Divergenz steht für das Gegenteil, nämlich eine wachsende Varianz der außenwirtschaftlichen Profile.

In der ausufernden Globalisierungsliteratur besteht für die Konvergenzerwartung auch das polemische Bild der "McDonaldization“, wie es Ritzer (1993) für kulturelle und gesellschaftliche Annäherungsprozesse geprägt hat. Nach der Auffassung eines anderen Globalisierungspopulisten geht die Uniformität aufgrund eines "dreifachen" Konvergenzprozesses so weit, dass man sich die Welt heutzutage wieder als Scheibe vorstellen könne (Friedman 2005); zentral sei dabei die Rolle, welche die Öffnung der bisher geschlossenen Gesellschaften im postkommunistischen Raum und in Asien gespielt habe. ${ }^{2}$ Auch wenn diese Literatur auf das Aufholen Asiens verweist, versteht sie Globalisierung primär als gemeinsame Annäherung an weltumspannende Wettbewerbsbedingungen, die für alle gleichermaßen gelten und keine unilateralen Anreize zum Austricksen der Konkurrenten schaffen.

Im Gegensatz zur Konvergenzthese steht die Erwartung einer neuen Divergenz, in der sich die Vorzeichen geändert haben. So gehen die Globalisierungsskeptiker von einem Wettlauf hin zu tiefer liegenden Regulierungsniveaus aus, in dem die einstmals autarken Staaten das Regulierungsniveau der relativ liberalisierten Länder unterbieten, um sich so im internationalen Standortwettbewerb besser behaupten zu können. Abrupte Öffnungen können Volkswirtschaften natürlich auch dermaßen überfordern, dass nur ein Teil der Staaten längerfristig mit radikalen Maßnahmen versucht, die Offenheit der liberalen Volkswirtschaften zu übertreffen. So haben besonders prominent Stiglitz (2002) und Rodrik (1992a, b, 2001) darauf hingewiesen, dass im Zuge der Globalisierung unter Umständen eher ein Auseinanderdriften als eine Annäherung der wirtschaftspolitischen Profile zu erwarten sei, da die Öffnung die ärmsten Länder überfordere.

Eine theoretische Grundlage für die Erwartung eines Regulierungswettbewerbs lässt sich in der Effizienzthese finden, die Sinn (1990) als einer der ersten formulierte und Garrett (1998a, b) popularisierte. Demnach zerbröckeln in einer Situation des globalen Wettbewerbs die Staatseinnahmen zusehends, da die mobilen Faktoren sich den widrigen Bedingungen, die in außenwirtschaftlich geschlossenen Ländern herrschen, entziehen können. Diese Anreizstruktur kann zu einem Regulierungswettbewerb beispielsweise in der Steuerpolitik führen. Der Unterbietungsprozess resultiert dann in zunehmender Divergenz, wenn die Liberalisierung in den einstmals restriktiven Staaten so stark ausfällt, dass sich zwischen den beiden Ländergruppen ein Abstand bildet, der größer ist als jener, der vor der Liberalisierung gegeben war. Konvergenz in Zeiten des Regu-

2 Friedman (2005) begreift Globalisierung vorwiegend als Konsequenz von technologischem Fortschritt. Er sieht Konvergenz vor allem in der Schaffung eines globalen, internet-basierten Marktplatzes, einer Enthierarchisierung und dem Entstehen neuer Möglichkeiten durch einen weltumspannenden Wettbewerb. 
lierungswettbewerbes liegt dann vor, wenn dieser Abstand geringer ausfällt als am Anfang der Globalisierung.

Vergessen wurde bis jetzt im Globalisierungsdiskurs, dass die Divergenz auch dann zunehmen kann, wenn sich der Globalisierungsclub vornehmlich auf jene Länder erstreckt, die schon vor den 1970er Jahren und damit vor der zweiten Welle der Globalisierung außenwirtschaftlich relativ offen waren. In einer solchen Welt der unterschiedlichen Öffnungsgeschwindigkeiten könnten sich die bislang zwar relativ autarken Volkswirtschaften ebenfalls zunehmend dem Wettbewerb öffnen. Dies geschieht aber nicht in einem derartigen Ausmaß, dass die Varianz über alle Länder gesehen im Laufe der Zeit abnimmt, weil die bereits relativ liberalen Länder ihr Regulierungsniveau weiter absenken. Dieses Argument lässt sich mit Arbeiten von Dani Rodrik, besonders dem mit Mukand verfassten Aufsatz verknüpfen (Mukand/Rodrik 2005).

Die meisten frühen Arbeiten zum "Wettlauf zum Freihandel“ (Rodrik 1992b) verweisen auf die innenpolitischen Triebfedern dieser Prozesse (Bodenstein 2005; Bodenstein/Schneider 2006; Martin 2005). In diesem Aufsatz lenken wir den Blick auf die internationale Dimension, die der vermuteten Annäherung der außenwirtschaftlichen Profile zugrunde liegt. Simmons und Elkins (2004; siehe auch Elkins u. a. 2006) haben zu Recht darauf hingewiesen, dass rein innenpolitische Modelle der Liberalisierung nicht genügen können. Zu diesem Zweck verstehen sie die nationalstaatliche Öffnung der Außenwirtschaftspolitik unter anderem auch als Antwort auf die Erfahrungen, welche soziokulturellen Vorbildsstaaten gemacht haben. Wir gehen ähnlich wie Simmons und Elkins (2004) davon aus, dass die Außenwirtschaftspolitik eine strategische Komponente aufweist und die Staaten durch die Öffnungsentscheidungen anderer Nationen unter Umständen unter Zugzwang geraten. ${ }^{3}$ Anders als Simmons und Elkins (2004) fokussieren wir dabei ausschließlich auf den Abhängigkeiten, die aus den Handelsbeziehungen zwischen Staaten - teilweise gepaart mit geographischer Nähe - erwachsen können.

Um die aus dieser Interdependenz entstehende Dynamik verstehen zu können, ergründen wir mit Hilfe eines einfachen Modells, unter welchen Bedingungen eher Konvergenz oder Divergenz zu erwarten ist. Ferner erlaubt das Modell Hinweise auf die Umstände, in welcher Situation ein Staat das Regulierungsniveau unterbieten wird (regulativer Wettbewerb) und wann der Öffnungsbeitrag des autarkeren Landes kleiner ausfällt als jener des bereits liberalisierten Landes (Pfadabhängigkeit). ${ }^{4}$ In Anlehnung an Holzer und Schneider (2002) betrachten wir die Interaktion zweier Staaten, die im Kontext einer exogen gegebenen weltweiten Offenheit das Niveau ihrer Außenwirtschaftsregulierung bestimmen. Unser Modell ähnelt dabei auf den ersten Blick dem Zwei-Akteur-Modell, das Drezner (2005) vorgelegt hat. So gehen wir in Übereinstimmung zu diesem Koordinationsspiel davon aus, dass Staaten ein Interesse an der Koordination ihrer Politik haben. Der entscheidende Unterschied zwischen den beiden Mo-

3 Interessanterweise differieren die Resultate von Simmons und Elkins (2004) und Elkins u. a. (2006). Während die erste Studie in der Analyse der generellen Liberalisierung der Außenwirtschaftspolitik auf die Erfahrungen in kulturell nahe stehenden Ländern verweist, ist in der zweiten Untersuchung, die sich mit der Verbreitung von bilateralen Investitionsverträgen beschäftigt, die Erklärungskraft solcher "konstruktivistischer" Variablen nur reduziert vorhanden.

4 Wie erwähnt, kann regulativer Wettbewerb mit Divergenz und Konvergenz verknüpft sein. 
dellen liegt aber im Autonomiegrad, den die Regierungen haben. So gehen wir von opportunistischen Regierungen aus, die in ihren Regulierungsentscheidungen im Sinne der Theorie des kollektiven Handelns auch die Leistungen anderer Staaten in ihr Kosten-Nutzen-Kalkül einbeziehen können. Drezner (2005) untersucht dagegen, inwiefern Staaten in Zeiten der Globalisierung zu einer verstärkt formalisierten Zusammenarbeit fähig sind. ${ }^{5}$

Die weltweite Offenheit $\left(\mathrm{O}_{\mathrm{t}}\right)$ lässt sich als Summe der einzelstaatlichen Offenheir $\left(o_{i, t}\right)$ darstellen, so dass

$O_{t}=\sum_{i=1}^{n} o_{i, t}$

Wir gehen davon aus, dass eine Veränderung der exogen gegebenen weltweiten Offenheit den Anreiz beider Staaten erhöht, ihre Außenwirtschaftspolitik zu verändern. Dies kann z. B. dadurch zu Stande kommen, dass mit einem Anstieg der weltweiten Offenheit die Opportunitätskosten einer restriktiven Regulierung zunehmen. Populär ausgedrückt: Die beiden Staaten, die wir in unserem Modell in Betracht ziehen, geraten unter einen "Globalisierungsdruck“.

Der einzelstaatliche Anteil an der Offenheit ist vom Grad der Offenheit in der Vorperiode, vom eigenen Regulierungsniveau $\mathrm{r}_{\mathrm{i}, \mathrm{t}}$ und dem Regulierungsniveau des Referenzstaates $r_{j, t}$ abhängig:

$o_{i, t}=f\left(o_{i, t-1}\right) r_{i, t}, r_{j, t}$, wobei $\frac{\partial o_{i, t}}{\partial o_{i, t-1}}>0, \frac{\partial o_{i, t}}{\partial r_{i, t}}<0, \frac{\partial o_{i, t}}{\partial r_{i, t}}>0$.

Die in Gleichung (2) dargestellte Funktion besagt, dass einzelstaatliche Offenheit aufgrund von exogenen Faktoren wie technischem Fortschritt und sinkenden Transportkosten ein positiv autoregressiver Prozess ist. In anderen Worten: Außenwirtschaftliche Öffnung folgt einem „Fluch der guten Tat", in dem eine Liberalisierung eine weitere Liberalisierung nach sich zieht. Je höher nun aber das eigene Regulierungsniveau ist, desto geringer ist der Anteil des Globalisierungsdrucks, den ein Land selber schultern muss. Umgekehrt hält Gleichung (2) auch die Anreize zum Regulierungswettbewerb fest, in dem die Liberalisierung eine positive Funktion der Regulierungsniveaus anderer Staaten ist. Oder anders ausgedrückt, der Globalisierungsdruck, der auf eine einzelne Volkswirtschaft wirkt, ist größer, wenn Referenzstaaten geschlossen sind, da sich Vorreiter nicht auf Vorleistungen dieser möglichen Vorbilder verlassen können. Die Modellannahme ist in Einklang mit dem Befund der empirischen Forschung, dass eine ausgeprägte Erhöhung des wirtschaftlichen Integrationsniveaus mit verstärktem Widerstand der Bevölkerung in Form von Streiks (Bussmann u. a. 2006) oder auch, zumindest in Afrika, Bürgerkriegen verbunden ist (Bussmann/Schneider 2007; Bussmann u. a. 2005).

5 Auf mehr technischer Ebene ist zu vermerken, dass in unserem Modell die Nutzenfunktionen der Akteure allgemeiner spezifiziert sind, so dass im Sinne der von uns in Betracht bezogenen Möglichkeiten - Wettbewerb, Konvergenz, Divergenz, Pfadabhängigkeit - reichhaltigere Ergebnisse zu erwarten sind. 
Wir definieren die Veränderung der Regulierung als $\delta_{\text {it }}$, wobei ein negativer Wert für eine Liberalisierung, ein positiver Wert für eine Abschottung vom Weltmarkt steht. Als verpflichtend gilt aber, dass die internationalen Normen, wie sie etwa für WTOMitglieder vorgegeben werden, nicht überschritten werden. Das heißt, dass das neue Regulierungsniveau unter dem Schwellenwert $\theta$ liegen muss. Diese Bedingung erfasst den Umstand, dass das Niveau der außenwirtschaftlichen Regulierung auch durch internationale Organisationen und international vereinbarten Normen beeinflusst ist.

Die Kosten der Regulierung hängen von der gegenwärtigen Offenheit und den Bedingungen des jeweiligen politischen Systems ab:

$c\left(o_{i, t}\right)=o_{i, t}$ und $c\left(\delta_{i, t}\right)=c_{i} \delta_{i, t}$, wobei $o_{i, t}=o_{i, t-1}\left(1+r_{i, t}\right)\left(1-r_{i, t}\right) \forall i \neq j$

Das Optimum der einzelstaatlichen Offenheit lässt sich aufgrund der vorherigen Ausführungen über diese Kostenfunktion unter zwei Nebenbedingungen berechnen. Zum einen ist zu beachten, dass die über internationale Verträge festgeschriebene Restriktivität nicht überschritten wird. Zum anderen müssen die Staaten den Globalisierungsdruck unter sich aufteilen. Für das Land i nimmt das Optimierungsproblem folgende Form an:

$L:=\frac{O}{2}\left(1+r_{i, 0}+\delta_{i, 1}\right)\left(1-r_{j, 0}-\delta_{j, 1}\right)+c_{i} \delta_{i, 1}+\lambda\left[O-o_{i}-o_{j}\right]+w\left[\theta-r_{i}\right]$

Die Ableitung von Gleichung (4) nach $\delta_{i, t}, \lambda$ und $\omega$ führt zu drei Gleichungen, aus denen sich die optimale Veränderung der Regulierung $\delta_{i, 1}^{*}$ berechnen lässt.

$\frac{\partial L}{\partial \delta_{i, 1}}=\frac{O}{2}\left(1-r_{j, 0}-\delta_{j, 1}\right)+c_{i}=0$

$\frac{\partial L}{\partial \lambda}=0-o_{i}-o_{j}=0$

$\frac{\partial L}{\partial \omega}=\theta-r_{i, 0}-\delta_{1,1} \geq 0$

Daraus abgeleitet ergibt sich aufgrund des symmetrischen Entscheidungsproblems die optimale Restriktionsänderung.

$\delta_{i, 1}^{*}=\frac{2\left(c_{j}-c_{i}\right)}{O}+\left(r_{j, 0}-r_{i, 0}\right)+\delta_{j, 1}$

Wenn nun Globalisierung jenes Schicksal ist, dem man sich nicht entziehen kann, hängt die Restriktionsänderung vom Verhältnis der Kosten und vom Regulierungsniveau in der Vorperiode ab. Für die nachfolgende Analyse schließen wir dabei Symmetrieannahmen aus, in denen die Kosten der Anpassung c oder der Restriktivitätsgrad der beiden Länder genau gleich groß sind. Tabelle 1 fasst die Hauptergebnisse unserer Analyse für das Zweiländermodell zusammen. 
Tabelle 1: Konvergenz und Divergenz in der Außenwirtschaftspolitik als Konsequenz unterschiedlicher Kostenstrukturen und ursprünglicher Regulierungsniveaus

\begin{tabular}{|c|c|c|}
\hline & $\begin{array}{c}\text { Staat } 1 \text { mit höheren } \\
\text { Anpassungskosten }\end{array}$ & $\begin{array}{c}\text { Staat 1 mit niedrigeren } \\
\text { Anpassungskosten }\end{array}$ \\
\hline Staat 1 ist protektionistischer & $\begin{array}{c}\text { Konvergenz oder } \\
\text { regulativer Wettbewerb }\end{array}$ & Unbestimmt \\
\hline Staat 1 ist freihändlerischer & Unbestimmt & Pfadabhängigkeit \\
\hline
\end{tabular}

Welche Szenarien plausibel sind, lässt sich mit Hilfe der komparativen Statik ergründen. Im Folgenden präsentieren wir die Ergebnisse einer numerischen Simulation. Abbildung 1 zeigt die optimale Veränderung der Regulierungsrestriktivität eines Landes, das sich mit einer Liberalisierung eines anderen Landes konfrontiert sieht. Simuliert wird die optimale Antwort des Landes über einen Parameterbereich, der alle möglichen Kombinationen von Kosten und ursprünglichem Regulierungsniveau abbildet. So be-

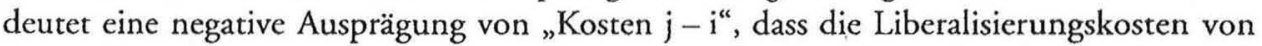
Land $i$ jene von Land $j$ übersteigen. Diese Interpretation gilt analog für das Regulierungsniveau. Wenn diese beiden Parameter negativ ausgeprägt sind, besteht für Land $i$ die optimale Reaktion auf eine Liberalisierung in $j$ in einer Anpassung der eigenen außenwirtschaftlichen Restriktivität nach unten.

Abbildung 1: Simulation der optimalen Öffnung in Abhängigkeit der relativen Kosten und des relativen Regulierungsgrades

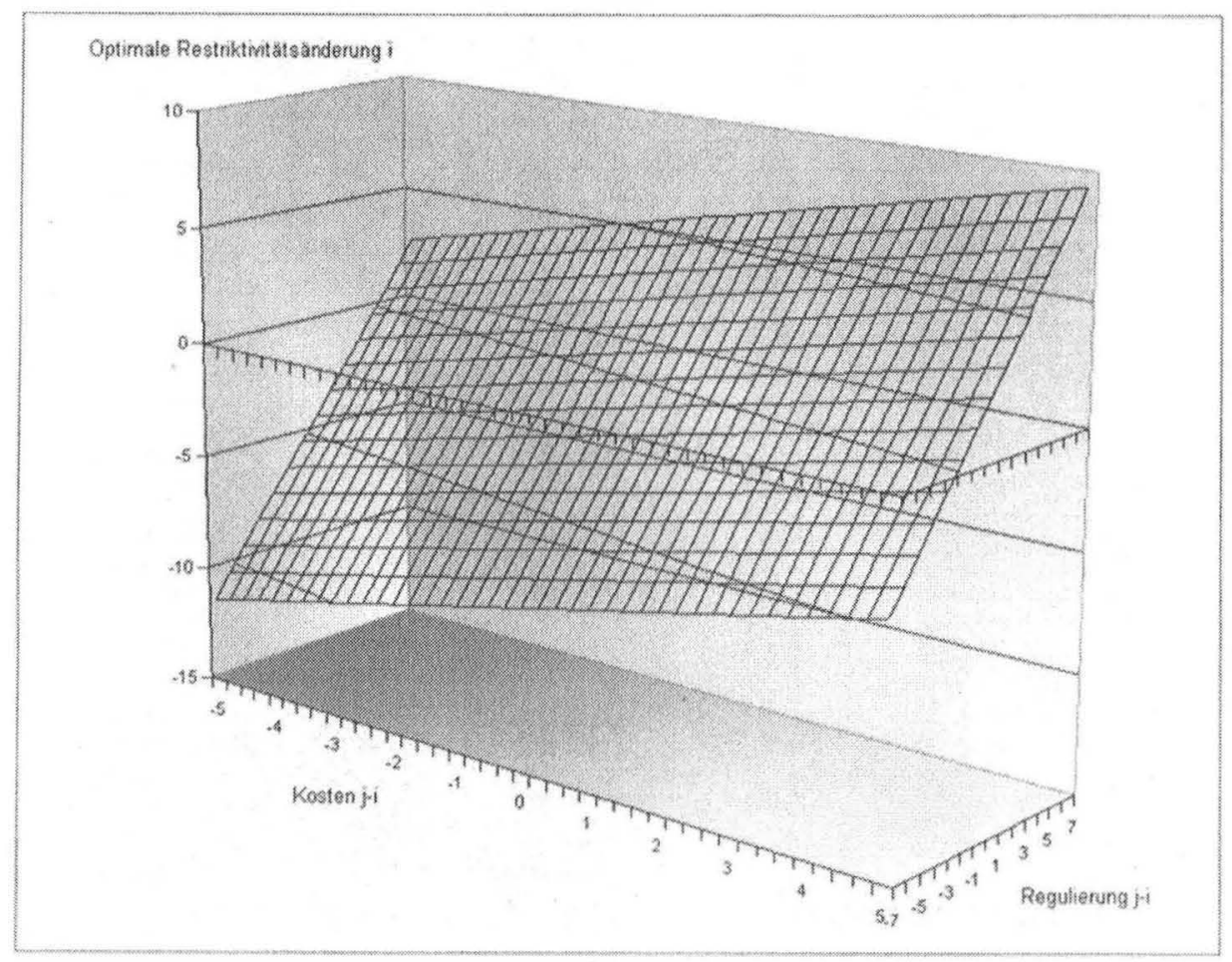


Die Analyse zeigt, dass Veränderungen der internationalen Wirtschaftspolitik vielfältige Formen annehmen können. Ein regulativer Wettbewerb - ausgedrückt durch einen sehr hohen Öffnungsbeitrag - ist dabei die wohl unwahrscheinlichste Form, wie Staaten mit dem Globalisierungsdruck umgehen. Ein solcher Wettbewerb bedeutet nämlich, dass die protektionistischen Staaten sich nicht nur stärker als die freihändlerischen Länder öffnen, sondern dass der Restriktionsabbau so stark ausfällt, dass sie auf einem tieferen Regulierungsniveau liegen als in der Vorperiode. Viel wahrscheinlicher ist es, dass das System konvergiert oder dass sich besonders die bereits relativ offenen Volkswirtschaften stärker öffnen als die autarken. Dies ist ebenfalls in der Graphik ersichtlich, wo vergleichsweise geringe Liberalisierungskosten mit einem höheren Öffnungsbeitrag korrelieren. In diesem Zusammenhang sprechen wir von einer pfadabhängigen Entwicklung im internationalen Öffnungsprozess. Globalisierung folgt in dieser Perspektive nur in Extremsituationen der Logik des Gefangenendilemmas, das die Nationalstaaten zu einem gegenseitigen Wettlauf zwingt. Ferner ist auch nicht zwangsläufig mit einer uniformen Konvergenz zu rechnen, nach der die bereits integrierten Staaten kleine Öffnungsschritte nehmen, während sich die protektionistischen Volkswirtschaften dieser OECD- oder IWF-Norm fügen und für ihre Verhältnisse dramatische Öffnungsleistungen vorzeigen.

Wie weit sich Staaten liberalisieren, hängt weitgehend von den Regulierungskosten ab. Obwohl beispielsweise von einer Handelsliberalisierung eine Steigerung der gesamtgesellschaftlichen Wohlfahrt erwartet werden kann, werden die Verlierer einer Öffnung des Handelsregimes versuchen, eine solche Veränderung der Politik zu verhindern. Für die vorliegende Fragestellung ist es dabei unerheblich, ob die Grenze zwischen Gewinnern und Verlierern entlang unterschiedlichen Sektoren oder entlang der Produktionsfaktoren verläuft. Wesentlich in der vorliegenden Betrachtung ist die theoretische Erwartung, wonach kein eindeutiger Trend in Richtung von Konvergenzprozessen zu erwarten steht. Wir gehen aber davon aus, dass der innenpolitische Widerstand gegen eine Handelsliberalisierung größer sein sollte als gegenüber eine Öffnung der Kapitalmärkte, da die innenpolitischen Anpassungskosten für eine Handelsöffnung sehr viel größer sind. Der eine Grund besteht darin, dass der Kreis der direkten Profiteure solcher Maßnahmen sehr klein ist. Zweitens ist in Rechnung zu stellen, dass ein Abbau von Kapitalkontrollen ein Land in kurzer Zeit attraktiv für ausländische Investoren machen kann. Mit anderen Worten: falls es zum regulativen Wettbewerb kommt, ist eher bei der Öffnung des Kapitalmarktes damit zu rechnen als bei der Liberalisierung der Handelspolitik. Im nächsten Abschnitt beschreiben wir, wie wir die verschiedenen theoretischen Erwartungen auf ihre Stichhaltigkeit hin empirisch überprüfen wollen.

\section{Forschungsanlage}

Dieser Aufsatz untersucht, ob regulativer Wettbewerb, Konvergenz oder Pfadabhängigkeit die Dynamik jenes Prozesses beschreiben, den wir gemeinhin als weltwirtschaftliche Integration oder "Globalisierung" bezeichnen. Wir greifen zur Überprüfung der unterschiedlichen Thesen zu den Mitteln der deskriptiven und der schließenden Statistik. Dabei ist grundlegend zwischen zwei Arten von Schätzungen zu unterscheiden: Im 
ersten Typ von Modellen bildet das Niveau der Handelsrestriktivität bzw. der Restriktivität der Kapitalverkehrsregulierung die abhängige Variable. Für diese Art von Schätzungen verwenden wir einen Datensatz, der für die Staaten im Sample die Beobachtungen über die Zeit verzeichnet. Die Daten stellen damit eine Kombination von Längschnitt- und Querschnittsdaten dar, wie sie in der Vergleichenden Politikwissenschaft seit Jahren üblich ist. Der zweite Typ von Modellen verwendet die Veränderung der Handelsrestriktivität bzw. der Restriktivität der Kapitalverkehrsregulierung zwischen 2002 und der zeitlich ersten Beobachtung (i. d. R. 1978) als abhängige Variable. Wir verwenden für diesen Typ von Modellen einfache OLS-Regressionen.

Abhängige Variable: Wir greifen für die empirische Überprüfung der vorgetragenen $\mathrm{Hy}$ pothesen auf einen neu verfügbaren, gegenüber Martin (2005) aufdatierten Datensatz zurück, der die Außenwirtschaftspolitik von 110 Staaten von 1978 bis 2002 abbildet. $^{6}$ Dabei wird zwischen den beiden Dimensionen "Internationaler Handel" (Güter und Dienstleistungen), sowie "Internationaler Kapitalverkehr" unterschieden. Die Variable für die Handelsdimension variiert zwischen 0 (völlige Freiheit) und 7 (hoch restriktive Regulierung); jene für den Kapitalverkehr zwischen 0 und 5 mit analogen Bedeutungen. Der Datensatz wurde auf der Grundlage des „Report on Exchange Arrangements and Exchange Restrictions" erstellt, den der Internationale Währungsfonds (IWF) jährlich veröffentlicht. In diesen Reports finden sich nach Ländern gegliedert zahlreiche Angaben zur Ausgestaltung der internationalen Wirtschaftsbeziehungen der Mitgliedstaaten des IWF. Die Codierung der IWF-Berichte resultierte in einem Datensatz, der über 50 numerische Ausgangsvariablen enthält. Diese wurden zu den beiden o. g. Maßen der Außenwirtschaftspolitik aggregiert (für Einzelheiten zum Datensatz vgl. Martin 2005).

Für die vorliegende Fragestellung stellen die beiden Indikatoren insofern geeignete endogene Variablen dar, als sich in ihnen explizit Politiken und keine Politikergebnisse abbilden. Dies unterscheidet die verwendeten Maße von anderen Indikatoren außenwirtschaftlicher Offenheit, wie beispielsweise dem in der Literatur häufig verwendeten Offenheitsmaß von Jeffrey Sachs und Andrew Warner (1995). Überdies existiert nach unserem Wissen kein Maß, das die Außenwirtschafsorientierung von derart vielen Ländern über einen vergleichbar langen Zeitraum im geforderten Detail abbildet.

Unabhängige Variablen. Als Kovariaten werden zum einen Faktoren in die Schätzungen aufgenommen, die in der Literatur als Determinanten der Außenwirtschaftspolitik diskutiert werden. Hierzu zählen neben dem durchschnittlichen Pro-Kopf-Bruttoinlandsprodukt als Maß für den Reichtum eines Landes eine Kenngröße für die Bedeutung des einheimischen Marktes (Bruttonationaleinkommen), zwei Indikatoren für den möglichen Einfluss makroökonomischer Krisen (Inflation und Wirtschaftswachstum), sowie als Proxy für das generelle Ausmaß staatlicher Eingriffe in Marktprozesse der Staatskonsum als Anteil am Bruttoinlandsprodukt. Diese Variablen stammen aus den "World Development Indicators 2004" der Weltbank (2004).

Unser Hauptinteresse richtet sich auf den Einfluss dreier Variablen, die wir in unterschiedlichen Kombinationen in den Schätzungen verwenden: die Restriktivität der

6 Eine Liste der Staaten im Sample findet sich im Anhang. 
Regulierung zu Beginn des Beobachtungszeitraums, die (unterschiedlich spezifizierte) Restriktivität der Regulierung in anderen Staaten, sowie der Mitgliedschaft im Internationalen Währungsfonds unter den Vorkehrungen des Artikel VIII IWF-Statut. Im Folgenden diskutieren wir kurz die Bedeutung und Operationalisierung dieser drei Variablen:

- Restriktivität der Regulierung in to: Diese Variable erfasst das Restriktivitätsniveau in einem Land zu Beginn des Beobachtungszeitraums. Der zu schätzende Koeffizient dieser Variable dient im Zusammenhang mit den Modellen, in denen das Niveau der Regulierung als abhängige Variable eingeht, als Indikator für die Bedeutung von pfadabhängigen Politikprozessen; für die Modelle der Veränderung von Regulierung wird sie als Indikator für die Stärke von Konvergenztendenzen verwendet.

- Restriktivität der Regulierung in anderen Staaten: Wir verwenden hier drei verschieden spezifizierte Variablen, die abbilden, welchen globalen oder regionalen Regulierungsniveaus sich ein Staat gegenübersieht: Erstens geht in die Schätzungen eine Variable ein, die die durchschnittlichen Restriktivität der Handels- bzw. Kapitalverkehrsregulierung erfasst. Die Variable errechnet sich als:

$$
A R_{i, t}=\frac{\sum_{j=1}^{N} R_{j, t}}{N}
$$

Dabei ist die durchschnittliche Restriktivität für Land i zum Zeitpunkt t, $A R_{\text {is }}$ das arithmetische Mittel aus der Summe der Regulierungsniveaus $R$ in den Ländern $j$ und $\mathrm{i} \neq \mathrm{j}$. Die Variable erfasst somit den durchschnittlichen Regulierungsgrad aus der Perspektive jenes Landes, für dessen eigene, davon rechnerisch unabhängige Regulierung der Einfluss des durchschnittlichen Regulierungsgrades und anderer Faktoren geschätzt werden soll. Diese Variable nimmt damit die im theoretischen Modell beschriebenen Interdependenzbeziehungen zwischen einzelnen Staaten auf. Einen genaueren Test der Theorie, wonach die Restriktivitätsniveaus im Fokus-Staat von der Restriktivität in anderen Staaten mit bestimmt werden, ermöglicht, zweitens, eine Variable, die die gewichtete Summe der Handelsrestriktivität anderer Länder erfasst. Die Gewichtung erfolgt durch $\mathrm{N} \times \mathrm{N}$-Gewichtungsmatrizen, die für die einzelnen Jahre im Beobachtungszeitraum getrennt berechnet werden. Die Gewichte liefern die bilateralen Handelsvolumina, die für die einzelnen Länder reihenstandardisiert werden. Das Konzept der Gewichtungsmatrizen stammt aus Arbeiten zur räumlichen Ökonometrie, für die Anselin (1988) einen grundlegenden Beitrag verfasst hat. Für die vorliegende Schätzung wurde die Gewichtung durch geografische Faktoren (z. B.: Distanz zwischen Hauptstädten, gemeinsame Grenze) durch die Bedeutung des bilateralen Handels ersetzt. Die Handelsrestriktivität eines Staates $j$ wird damit umso wichtiger, je mehr der Fokusstaat i mit diesem Staat handelt. Ist zwischen zwei Staaten kein Handel zu beobachten, so wird die Handelsrestriktivität nicht berücksichtigt. Trotz der Endogenitätsprobleme, die einem solchen Test inhärent sind, erfasst diese Spezifikation die Anreize und Hemmnisse zu einem Wandel in der Handelspolitik wesentlich genauer, als dies durch die Berücksichtung bloßer Durchschnittswerte möglich ist. Weil bilaterale Daten nur für Handelsbeziehungen, nicht aber für 
den Kapitalverkehr verfügbar sind, kann diese Variable nur in den Modellen eingesetzt werden, die Determinanten der Handelsrestriktivität schätzen. Schließlich berücksichtigen wir, drittens, die Bedeutung regionaler Einflüsse, indem wir in einer Gewichtungsmatrix die Handelsvolumina durch Dummy-Variablen ersetzen, die den Wert 1 annehmen, wenn sich zwei Staaten in derselben Region befinden. Wir unterscheiden an dieser Stelle zwischen den Regionen "Lateinamerika und Karibik“, „Afrika südlich der Sahara“, „Nordafrika und Mittlerer Osten“, sowie „Asien“. Außerdem führen wir eine nicht geografisch definierte Region „OECD“ ein, in der sich alle Staaten befinden, die zum Beobachtungszeitpunkt Mitglied der OECD waren. Ein Staat ist jeweils nur einer Region zugeordnet, wobei das Kriterium „OECD“ Vorrang genießt. Die regionengewichtete Restriktivitätsvariable ist sowohl für die Handels- als auch für die Kapitalverkehrsregulierung verfügbar.

- Art VIII IWF-Statut: Dieser Artikel erlegt den Staaten, die ihn unterzeichnet haben, eine Reihe von Verpflichtungen auf, die unmittelbar mit der staatlichen Regulierung des internationalen Handels und des internationalen Kapitalverkehrs verknüpft sind. Signatarstaaten ist es abgesehen von wenigen Ausnahmen untersagt, in die Freiheit von Handel und Zahlungsverkehr einzugreifen. Andererseits bietet der IWF seinen Mitgliedsstaaten mit den alternativen Vorkehrungen des Artikel XIV die Möglichkeit, ihre Außenwirtschaftspolitiken restriktiver zu gestalten. Diese Vorkehrungen gelten als vorläufig und vorübergehend und sind auf Staaten gerichtet, die sich noch nicht „reif" genug fühlen, ihre IWF-Mitgliedschaft unter den engeren Regularien des Artikels VIII auszugestalten. Wir verwenden diese Variable, um den Einfluss von internationalen Normen zu testen. Die anderen zentralen Variablen beziehen sich ebenfalls auf das formale Modell, berücksichtigen aber stärker die positiven Anreize.

\section{Die empirische Substanz dreier Globalisierungserklärungen}

Die akademische und populärwissenschaftliche Literatur hat eine Vielzahl von Hypothesen zum Prozess der außenwirtschaftlichen Öffnung hervorgebracht. In diesem Aufsatz überprüfen wir drei konkurrierende Thesen. Die erste postuliert, dass die autarken Staaten den Offenheitsgrad der liberalen Staaten zu unterbieten versuchen („regulativer Wettbewerb"); die zweite Hypothese geht davon aus, dass sich alle Staaten öffnen, aber der Wettbewerb um geeignete Standortbedingungen insgesamt eine geringe Rolle spielt („Konvergenz"), und die dritte Behauptung geht insofern von unterschiedlichen Globalisierungsgeschwindigkeiten aus, als die Öffnung vorwiegend in den bereits relativ freihändlerischen Staaten erfolgen soll („Pfadabhängigkeit“).

In Einklang mit der Literatur möchten wir dabei zunächst in deskriptiver Weise überprüfen, ob die Daten Hinweise auf eine globale Konvergenz der Außenwirtschaftspolitiken liefern. Die Standardabweichung der Regulierungsniveaus ist eines der üblichen Maße, mit denen sich die Dynamik der Globalisierung erfassen lässt. ${ }^{7}$ Ein Rückgang der Standardabweichung ist eine notwendige und hinreichende Bedingung für die Konvergenz der Regulierungen. Abbildung 2 zeigt die Entwicklung der Standardabwei-

7 Die Verwendung von Variationskoeffizienten liefert im Wesentlichen die gleichen Ergebnisse. 
chung über den Beobachtungszeitraum 1978 bis 2002 für die ca. 110 Staaten in unserem Sample ${ }^{8}$. Für beide Arten der Außenwirtschaftsregulierung zeigt sich ein moderater Anstieg der Standardabweichungen, so dass vor diesem Hintergrund nicht von einer Konvergenz der Politiken ausgegangen werden kann. Im Gegenteil regulierten die Länder im Sample ihre Außenwirtschaftsbeziehungen am Ende der Beobachtungsperiode unterschiedlicher als zu deren Beginn.

Abbildung 2: Standardabweichungen für zwei Dimensionen außenwirtschaftlicher Offenheit 1978-2002

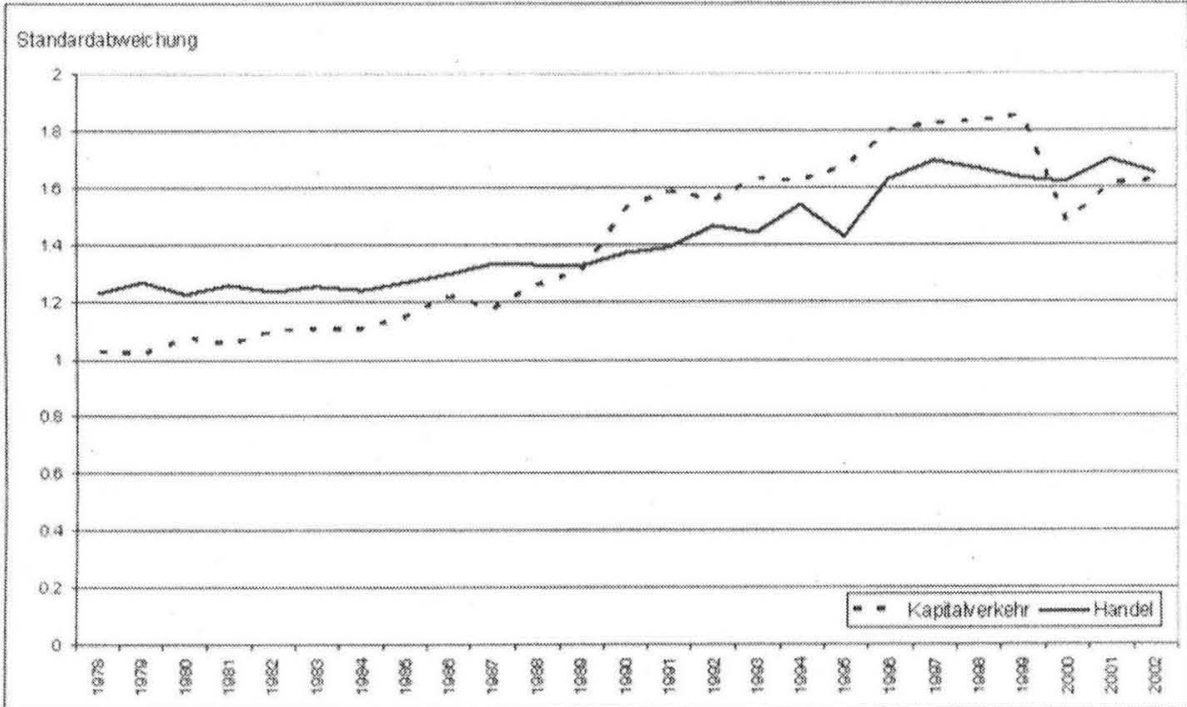

Allerdings verstellt die aggregierte Betrachtung über alle Staaten den Blick auf mögliche Konvergenzprozesse, die sich innerhalb einzelner Sub-Samples ergeben haben mögen. So sank beispielsweise die Standardabweichung des Maßes für die Restriktivität der Außenhandelsregulierung in OECD-Staaten von $1.35 \mathrm{im}$ Jahr 1978 auf $0.71 \mathrm{im}$ Jahr 1999. Im gleichen Zeitraum stieg sie aber in den übrigen Ländern von 1.16 auf 1.75. Konvergenz bleibt damit auf einzelne Staatengruppen beschränkt. Diese Entwicklungen liefern erste Hinweise darauf, dass die Globalisierung zumindest in der Dimension der Außenwirtschaftspolitik sehr ungleich verlaufen ist.

Klare Aussagen zur Gültigkeit unserer Hypothesen können aber nur multivariate Analysen liefern. Tabelle 2 zeigt die Ergebnisse einer multivariaten Schätzung, in der das Niveau der Restriktivität der Handelsregulierung die abhängige Variable bildet. Wir schätzen eine Prais-Winsten-Regression mit panelspezifischen autoregressiven Prozessen. In die Grundschätzung gehen neben der Handelsrestriktivität zu Beginn des Beobachtungszeitraums die oben diskutierten Kontrollvariablen ein. Modell 1 berücksichtigt außerdem die durchschnittliche Außenhandelsrestriktivität in anderen Ländern; in Mo-

8 Die Zahl der Staaten im Sample variiert über den Beobachtungszeitraum von 103 (1978) bis 118 (2002). 
dell 2 geht als ein Maß für den Einfluss internationaler Normen die Mitgliedschaft unter Artikel VIII IWF-Statut ein. Schließlich schätzt Modell 3 alle Einflussfaktoren gemeinsam. Die Modelle 4 und 5 nehmen die handelsgewichtete Restriktivität anderer Länder in die Schätzung auf, einmal mit Jahresdummies, einmal ohne. Das abschließende sechste Modell berücksichtigt die regional gewichtete Handelsrestriktivität als alternativen Erklärungsfaktor.

Tabelle 2: Empirische Schätzungen für Determinanten der Handelsrestriktivität

\begin{tabular}{|c|c|c|c|c|c|c|}
\hline & (1) & (2) & (3) & (4) & (5) & (6) \\
\hline Handelsrestriktivität $t_{0}$ & $\begin{array}{l}0.696^{* * *} \\
(10.45)\end{array}$ & $\begin{array}{l}0.624^{* * *} \\
(8.39)\end{array}$ & $\begin{array}{l}0.673^{* * *} \\
(10.21)\end{array}$ & $\begin{array}{l}0.618^{* * *} \\
(6.27)\end{array}$ & $\begin{array}{l}0.650^{* * *} \\
(9.05)\end{array}$ & $\begin{array}{l}0.677^{* * *} \\
(11.38)\end{array}$ \\
\hline $\begin{array}{l}\text { Durchschnittliches Pro. } \\
\text { Kopf-Einkommen (log) }\end{array}$ & $\begin{array}{l}-0.271^{* 0 .} \\
(-3.47)\end{array}$ & $\begin{array}{l}-0.163^{\circ} \\
(-1.82)\end{array}$ & $\begin{array}{l}-0.154^{* *} \\
(-2.02)\end{array}$ & $\begin{array}{l}-0.453^{* 6 .} \\
(-4.06)\end{array}$ & $\begin{array}{l}-0.317^{4 * 4} \\
(-3.88)\end{array}$ & $\begin{array}{l}-0.192^{* *} \\
(-2.28)\end{array}$ \\
\hline GNI $(\log )$ & $\begin{array}{r}0.049 \\
(1.12)\end{array}$ & $\begin{array}{l}0.068^{* *} \\
(2.15)\end{array}$ & $\begin{array}{c}0.036 \\
(0.86)\end{array}$ & $\begin{array}{l}0.116^{* * *} \\
(2.84)\end{array}$ & $\begin{array}{l}0.101^{* *} \\
(2.11)\end{array}$ & $\begin{array}{r}0,072 \\
(1.63)\end{array}$ \\
\hline $\log \operatorname{Inflation}(\operatorname{Lag} 1)$ & $\begin{array}{l}-0.004 \\
(-0.26)\end{array}$ & $\begin{array}{c}0.003 \\
(0.14)\end{array}$ & $\begin{array}{l}-0.004 \\
(-0.25)\end{array}$ & $\begin{array}{l}0.0009 \\
(0.04)\end{array}$ & $\begin{array}{c}0.002 \\
(0.09)\end{array}$ & $\begin{array}{r}0.007 \\
(0.49)\end{array}$ \\
\hline $\begin{array}{l}\text { Wirtschaftswachstum } \\
\text { (Lag 1) }\end{array}$ & $\begin{array}{l}0.128 \\
(0.65)\end{array}$ & $\begin{array}{l}0.314 \\
(1.22)\end{array}$ & $\begin{array}{r}0.109 \\
(0.55)\end{array}$ & $\begin{array}{r}0.002 \\
(0.79)\end{array}$ & $\begin{array}{r}0.004 \\
(1.55)\end{array}$ & $\begin{array}{r}0.003 \\
(1.21)\end{array}$ \\
\hline Staatskonsum (\% GDP) & $\begin{array}{l}-0.003 \\
(-0.45)\end{array}$ & $\begin{array}{r}0.003 \\
(0.48)\end{array}$ & $\begin{array}{l}-0.003 \\
(-0.44)\end{array}$ & $\begin{array}{l}0.005 \\
(0.59)\end{array}$ & $\begin{array}{r}-0.003 \\
(-0.38)\end{array}$ & $\begin{array}{l}0.0003 \\
(0.05)\end{array}$ \\
\hline $\begin{array}{l}\text { Durchschnituliche } \\
\text { Handelsrestriktivität } \\
\text { (alle lander) }\end{array}$ & $\begin{array}{l}1.024^{* * *} \\
(16.79)\end{array}$ & & $\begin{array}{l}0.902^{* * *} \\
(13.94)\end{array}$ & & & \\
\hline $\begin{array}{l}\text { IWF-Mitgliedschaft } \\
\text { unter Artikel VIII }\end{array}$ & & $\begin{array}{l}-0.862^{* * 8} \\
(-9.93)\end{array}$ & $\begin{array}{l}-0.414^{* * *} \\
(-5.40)\end{array}$ & & & \\
\hline $\begin{array}{l}\text { Handelsgewichrete } \\
\text { Handelsrestriktivitar } \\
\text { anderer Lander }\end{array}$ & & & & $\begin{array}{l}0,177^{* * *} \\
(3.44)\end{array}$ & $\begin{array}{l}0.080^{\circ *} \\
(2.23)\end{array}$ & \\
\hline $\begin{array}{l}\text { Regional gewichtere } \\
\text { Handelsrestriktivität } \\
\text { anderer LXnder }\end{array}$ & $\cdot$ & & , & & & $\begin{array}{l}0.443^{\text {k*x }} \\
(3.00)\end{array}$ \\
\hline Achsenabschnit & $\begin{array}{l}-3.067^{* 0} \\
(-4.25)\end{array}$ & $\begin{array}{l}1.581^{* *} \\
(1.99)\end{array}$ & $\begin{array}{l}-2.742^{* * *} \\
(-3.25)\end{array}$ & $\begin{array}{l}1.591^{*} \\
(1.82)\end{array}$ & $\begin{array}{l}1.702^{* *} \\
(2.46)\end{array}$ & $\begin{array}{l}-1.522 \\
(-1.14)\end{array}$ \\
\hline Jahresdummies & Nein & Nein & Nein & Nein & Ja & ja \\
\hline$R^{2}$ & 0.8604 & 0.8382 & 0.8415 & 0.7817 & 0.8737 & 0.8674 \\
\hline Wald -Chi ${ }^{2}$ & $881,40^{* * *}$ & $1056.68^{* * *}$ & $969.20^{k * *}$ & $609.50^{* * *}$ & $9559.00^{* * *}$ & $1079.77^{* * *}$ \\
\hline $\mathrm{N}$ & 1931 & 1931 & 1931 & 1650 & 1650 & 1920 \\
\hline Lander & 107 & 107 & 107 & 100 & 100 & 107 \\
\hline Jahre & $1980-2002$ & $1980-2002$ & $1980-2002$ & $1980-2000$ & $1980-2000$ & $1980-2002$ \\
\hline
\end{tabular}

Abhängige Variable ist die Restriktivität der Handelspolitik. Prais-Winsten-Regression mit panel-korrigierten Standardfehlern und panelspezifischen ARI-Prozessen im Fehlerterm, z-Statistiken in Klammern.

* signifikant auf Zehn-Prozent-Niveau, ${ }^{* *}$ signifikant auf Fünf-Prozent-Niveau, *** signifikant auf Ein-Prozent-Niveau. 
In allen Modellen besteht ein positiver Zusammenhang zwischen der Höhe des Regulierungsniveaus zu Beginn des Beobachtungszeitraums und dem Restriktivitätsgrad der folgenden Perioden: Je restriktiver ein Staat seine Außenhandelspolitik im Ausgangsjahr 1979 gestaltete, desto restriktiver war seine Regulierung in den Folgejahren. Dies legt einen pfadabhängigen Prozess nahe, innerhalb dessen die Abkehr von eingeschlagenen Politiken nur schwer möglich ist. Allerdings impliziert die Größe der Koeffizienten, die für alle Modelle unter 1 liegt, eine Reduktion der Handelsrestriktivität über die Zeit.

Für den Einfluss der Durchschnittsrestiktivität in anderen Staaten wird in den Modellen 2 und 4 ebenfalls ein positiver Zusammenhang geschätzt. Allerdings zeigt sich bei der Berücksichtigung der Norm-Variable in Modell 4 ein unterproportionaler positiver Zusammenhang zwischen der Restriktivität der Durchschnittsregulierung und dem Regulierungsniveau im Staat unter Beobachtung. Wir folgern daraus, dass internationalen Normen, zumindest in der vorliegenden Operationalisierung, ein liberalisierender Einfluss zuzusprechen ist. Dieser Einfluss zeigt sich sowohl im direkten negativen Zusammenhang zwischen der Dummy-Variable "Mitgliedschaft unter Artikel VII“" als auch indirekt in der Veränderung des Einflusses der Durchschnittsregulierung.

Die Variable, die die gewichtete Handelsrestriktivität anderer Staaten abbildet (Modell 4), ist im Einklang mit der Theorie positiv und signifikant und liefert einen Effekt in einer plausiblen Größenordnung. Dieser Effekt bleibt auch dann erhalten, wenn in einer konservativen Variante der Schätzung Dummy-Variablen für die einzelnen Jahre in das Modell mit aufgenommen werden (Modell 5). Zwar nimmt die Bedeutung des Effektes ab; ein gemeinsamer exogener Trend kann aber als alleinige Ursache für die in Modell 4 angestellten Beobachtungen ausgeschlossen werden.

In dem Modell 6 wird eine Variable in die Schätzung einbezogen, die die Handelsrestriktivität innerhalb einer Region abbildet. Diese Variable ist so konstruiert, dass die Handelsregulierung anderer Staaten nur dann ins Gewicht fällt, wenn diese anderen Länder sich in derselben Region wie der Fokus-Staat befinden. Auffällig bei der signifikanten und positiven Schätzung des Koeffizienten dieser Variable ist seine Größe: die Bedeutung der Regulierung innerhalb einer Region scheint jene der globalen Regulierung um ein Vielfaches zu übersteigen.

Ein im Wesentlichen gleiches Bild ergibt sich für die Verwendung der Kapitalverkehrsrestriktivität als abhängige Variable, zeigt doch Tabelle 3 auch für diese Dimension der außenwirtschaftlichen Regulierung einen positiven und signifikanten Einfluss der Ausgangsregulierung. Die Regulierung auf globaler und regionaler Ebene wird in allen Spezifikationen positiv und signifikant geschätzt. Offenbar kommt auch bei der Regulierung des Kapitalverkehrs der regionalen Ebene eine bedeutsame Rolle zu. Internationale Normen, die in Richtung einer Liberalisierung gefasst sind, zeigen auch bei der Kapitalverkehrsrestriktivität einen signifikanten und negativen Einfluss. Dies bedeutet, dass Staaten ihre Öffnung strategisch vollziehen und von der Politik von Referenzstaaten abhängig machen.

Abschließend wenden wir uns der Frage zu, ob wir in unserem Sample Konvergenzprozesse beobachten können. Wir verwenden als abhängige Variable in den Schätzungen, die in Tabelle 4 dargestellt sind, die Veränderung der Restriktivität der Außenwirtschaftsregulierung über den Beobachtungszeitraum. Modell 1 zeigt die Ergebnisse für die Veränderung der Handelsregulierung, Modell 2 schätzt den Einfluss der glei- 
Tabelle 3: Empirische Schätzungen für Determinanten der Kapitalverkehrsrestriktivität

\begin{tabular}{|c|c|c|c|c|}
\hline & (1) & (2) & (3) & (4) \\
\hline Kapitalverkehrstestriktivität $\mathrm{t}_{0}$ & $\begin{array}{l}0.768^{* * *} \\
(11.56)\end{array}$ & $\begin{array}{l}0.721^{\cdots *} \\
(13.64)\end{array}$ & $\begin{array}{l}0.737^{\cdots *} \\
(13.24)\end{array}$ & $\begin{array}{l}0.766^{* * *} \\
(9.24)\end{array}$ \\
\hline $\begin{array}{l}\text { Durchschnittliches } \\
\text { Pro-Kopf-Einkommen (log) }\end{array}$ & $\begin{array}{l}-0.2686^{* * *} \\
(-2.84)\end{array}$ & $\begin{array}{l}-0.264^{* *} \\
(-2.70)\end{array}$ & $\begin{array}{l}-0.225^{* *} \\
(-2.37)\end{array}$ & $\begin{array}{l}-0.200^{*} \\
(-1.93)\end{array}$ \\
\hline GNI (log) & $\begin{array}{l}-0.004 \\
(-0.08)\end{array}$ & $\begin{array}{r}0.021 \\
(0.67)\end{array}$ & $\frac{-0.007}{(-0.18)}$ & $\begin{array}{l}-0.016 \\
(-0.36)\end{array}$ \\
\hline $\log$ Inflation (Lag 1) & $\begin{array}{l}-0.003 \\
(-0.26)\end{array}$ & $\begin{array}{l}-0.009 \\
(-0.60)\end{array}$ & $\begin{array}{l}-0.001 \\
(-0.08)\end{array}$ & $\begin{array}{r}-0.009 \\
(-0.63)\end{array}$ \\
\hline $\begin{array}{l}\text { Wirtschaftswachstum } \\
\text { (Lag 1) }\end{array}$ & $\begin{array}{r}0.064 \\
(0.30)\end{array}$ & $\begin{array}{r}0.193 \\
(0.82)\end{array}$ & $\begin{array}{l}0.042 \\
(0.20)\end{array}$ & $\begin{array}{l}0.0001 \\
(0.09)\end{array}$ \\
\hline Staatskonsum (\% GDP) & $\begin{array}{l}-0.002 \\
(-0.40)\end{array}$ & $\begin{array}{l}0.006 \\
(1.13)\end{array}$ & $\begin{array}{l}0.0005 \\
(0.10)\end{array}$ & $\begin{array}{r}-0.002 \\
(-0.51)\end{array}$ \\
\hline $\begin{array}{l}\text { Durchschnittliche Kapitalverkehrs } \\
\text { restriktivität (alle Länder) }\end{array}$ & $\begin{array}{l}0.756^{* * *} \\
(13.47)\end{array}$ & & $\begin{array}{l}0.679^{* * *} \\
(11.30)\end{array}$ & \\
\hline $\begin{array}{l}\text { IWF-Mitgliedschaft unter } \\
\text { Artikel VIII }\end{array}$ & & $\begin{array}{l}-0.597^{* * *} \\
(-8.59)\end{array}$ & $\begin{array}{l}-0,323^{* * *} \\
(-4.70)\end{array}$ & \\
\hline $\begin{array}{l}\text { Regional gewichrete } \\
\text { Kapitalverkehrsrestriktivitär }\end{array}$ & & & & $\begin{array}{l}0.293^{* * *} \\
(2.06)\end{array}$ \\
\hline Achsenabschnitt & $\begin{array}{l}-0.051 \\
(-0.05)\end{array}$ & $\begin{array}{l}2.517^{* * *} \\
(4.49)\end{array}$ & $\begin{array}{r}0.185 \\
(0.22)\end{array}$ & $\begin{array}{l}68.828^{* * *} \\
(2.75)\end{array}$ \\
\hline Jahresdummies & Nein & Nein & Nein & Ja \\
\hline$R^{2}$ & 0.8237 & 0.8654 & 0.8215 & 0.8258 \\
\hline Wald-Chi ${ }^{2}$ & $1080.17^{\cdots *}$ & $1068.72^{* * *}$ & $1430.93^{* * *}$ & $627.69^{* * *}$ \\
\hline $\mathrm{N}$ & 1809 & 1806 & 1806 & 1804 \\
\hline Länder & 112 & 109 & 109 & 113 \\
\hline Jahre & $1980-2002$ & $1980-2002$ & $1980-2002$ & $1980-2002$ \\
\hline
\end{tabular}

chen Variablen auf die Veränderung der Kapitalverkehrsregulierung. Auch in diesen Schätzungen erweist sich der Einfluss der Normvariable für beide Dimensionen der Außenwirtschaftspolitik als liberalisierender Einfluss. Bedeutsamer erscheinen allerdings die negativen Vorzeichen für die beiden Koeffizienten, die den Einfluss des Regulierungsniveaus zu Beginn des Beobachtungszeitraumes abbilden. Eine restriktive Regulierung in to führt zu einer stärkeren Liberalisierung der Außenwirtschaftsbeziehungen. Dieser Effekt fällt bei der Kapitalmarktliberalisierung stärker aus als bei der Außenhandelspolitik, was auf eine verstärkte Bedeutung des Wettbewerbselementes in diesem Politikbereich hindeuten könnte. Wir interpretieren die negativen Koeffizienten für die beiden Variablen, die das Niveau der Ausgangsregulierung für Handel und Kapitalverkehr erfassen, als Hinweise auf $\beta$-Konvergenz. Dieser Konvergenzeffekt fällt allerdings über die einzelnen Staatengruppen hinweg zu uneinheitlich aus, um einen Prozess der $\sigma$-Konvergenz herbeizuführen, der die Standardabweichung im gesamten Sample senken würde. Unsere Beobachtungen für die unterschiedlichen Regionen legen vielmehr die Existenz von "Konvergenzclubs“ (Durlauf/Johnson 1995; Galor 1996) nahe, also einzelne Gruppen von Staaten, für die die Varianz der Regulierung abgenommen hat. 
Tabelle 4: Veränderung der Restriktivität der Außenwirtschaftsregulierung 1979-2002, OLS-Regression

\begin{tabular}{|lcc|}
\hline & $(1)$ & $(2)$ \\
\hline Restriktivität $t_{0}$ & $-0.059^{* *}$ & $-0.091^{* *}$ \\
Durchschnittliches Pro-Kopf-Einkommen (log) & $(-2.64)$ & $(-2.60)$ \\
& -0.047 & -0.021 \\
GNI (log) & $(-1.14)$ & $(-0.34)$ \\
& 0.018 & 0.023 \\
Log Inflasion & $(1.01)$ & $(0.94)$ \\
& $-0.038^{*}$ & -0.034 \\
Wirschaftswachstum & $(-1.81)$ & $(-1.18)$ \\
& 1.436 & 1.711 \\
Staatskonsum (\% GDP) & $(1.02)$ & $(0.74)$ \\
& -0.002 & $-0.015^{*}$ \\
IWF Mitgliedschaft unrer Artikel VIII (Jahre) & $(-0.38)$ & $(-1.90)$ \\
& $-0.010^{* * *}$ & $-0.014^{* *}$ \\
Achsenabschnitt & $(-2.85)$ & $(-2.61)$ \\
& $1.35278^{* * *}$ & $1.297^{* * *}$ \\
Adj. R2 & $(4.02)$ & $(2.69)$ \\
F.Stat & 0.1837 & 0.1596 \\
N (Länder) & $4.05^{* * *}$ & $3.33^{* * *}$ \\
\end{tabular}

\section{Fazit}

Die weltwirtschaftliche Integration stellt, auch wenn die Konjunktur des Begriffs der Globalisierung am Verebben ist, immer noch ein zentrales Forschungsgebiet innerhalb der Sozialwissenschaften dar. Trotz einer ständig wachsenden Zahl von Beiträgen wissen wir jedoch wenig darüber, wie einheitlich oder uneinheitlich Globalisierungsprozesse verlaufen. Dies hat besonders den zwei Thesen Auftrieb gegeben, wonach es sich bei der weltwirtschaftlichen Integration um einen weltweit umspannenden Konvergenzprozess handle oder aber um einen Regulierungswettbewerb, in dem geschlossene Staaten die Hürden, die dem freien Verkehr von Gütern und Kapital im Wege stehen, so schnell abbauen, dass sie offenere Staaten unterbieten können.

Dieser Aufsatz zeigt, dass aus einer theoretischen Perspektive auch unter Bedingungen einer zunehmenden Globalisierung kein einheitliches Muster der Außenwirtschaftspolitik zu erwarten ist. Konvergenz, Pfadabhängigkeit und regulativer Wettbewerb sind Prozesse, die in unterschiedlicher Mischung gleichzeitig in unterschiedlichen Gruppen von Ländern auftreten können. In unseren empirischen Tests fanden wir keine Hinweise auf die Existenz eines regulativen Wettbewerbs der Außenwirtschaftspolitik. Statt dessen erwiesen sich internationaler Normen als signifikant liberalisierender Effekt. Darüber hinaus fanden wir bei der Betrachtung der Veränderung von Außenwirtschaftspolitiken über die Zeit einen Trend zu Konvergenz, der aber nicht hinreicht, um die anhaltende und über das Gesamtsample gesehen steigende Divergenz von Außenwirt- 
schaftspolitiken zu kompensieren. Wir betrachten diese Beobachtung als Ausdruck des starken Einflusses pfadabhängiger Prozesse bei der Formulierung und Implementierung von Außenwirtschaftspolitik.

Ein logischer nächster Schritt in diesem Forschungsstrang besteht vor dem Hintergrund der hier vorgelegten Ergebnisse in einer genaueren Betrachtung der innerstaatlichen Determinanten der Außenwirtschaftspolitik. Ein vollständiges Modell der außenwirtschaftlichen Regulierung müsste beide Gruppen von Einflussfaktoren - innerstaatliche ebenso wie internationale - in den Blick nehmen und aufeinander beziehen. Ein solches Forschungsprogramm erscheint viel versprechend mit Blick auf alte Debatten in der Globalisierungsdiskussion.

\section{Anhang}

Länder im Sample und Zahl der Beobachtungen für zwei Dimensionen der Außenwirtschaftspolitik

\begin{tabular}{|lcc|}
\hline Land & N Handelsregulierung & N Kapitalverkehrsregulierung \\
\hline Agypten & 25 & 25 \\
Algerien & 25 & 25 \\
Angola & 14 & 14 \\
Argentinien & 22 & 25 \\
Arthiopien & 7 & 25 \\
Australien & 25 & 25 \\
Bangladesch & 25 & 25 \\
Belgien & 22 & 25 \\
Benin & 22 & 25 \\
Bhutan & 4 & 7 \\
Bolivien & 22 & 7 \\
Botswana & 6 & 13 \\
Brasilien & 25 & 25 \\
Burkina Faso & 22 & 22 \\
Burundi & 25 & 25 \\
Chile & 25 & 25 \\
Costa Rica & 4 & 5 \\
Danemark & 25 & 25 \\
Demokratische Republik Konge & 25 & 25 \\
Deurschland & 25 & 25 \\
Dominikanische Republik & 25 & 21 \\
Ecuador & 25 & 7 \\
El Salvador & 17 & 25 \\
Elfenbeinkuste & 24 & 24 \\
Eritrea & 1 & 7 \\
Finnland & 25 & 25 \\
Frankreich & 25 & 25 \\
Gabun & 21 & 25 \\
Gambia & 25 & 23 \\
Ghana & 25 & 25 \\
Griechenland & 15 & 25 \\
Großbritannien & 25 & 13 \\
Guatemala & 20 & 4 \\
& & \\
\hline
\end{tabular}




\begin{tabular}{|c|c|c|}
\hline Land & N Handelsregulierung & N Kapitalverkehrsregulierung \\
\hline Guinea & 24 & 25 \\
\hline Guinea-Bissau & 25 & 3 \\
\hline Haiti & 16 & 3 \\
\hline Honduras & 18 & 5 \\
\hline Indien & 18 & 25 \\
\hline Indonesien & 25 & 25 \\
\hline Iran & 25 & 25 \\
\hline Irland & 25 & 25 \\
\hline Island & 25 & 25 \\
\hline Italien & 25 & 25 \\
\hline Jamaika & 19 & 25 \\
\hline Japan & 25 & 25 \\
\hline Jemen & 25 & 25 \\
\hline Jordanien & 7 & 25 \\
\hline Kambodscha & 3 & 7 \\
\hline Kamerun & 4 & 25 \\
\hline Kanada & 25 & 25 \\
\hline Kenia & 20 & 25 \\
\hline Kolumbien & 25 & 25 \\
\hline Laos & 19 & 10 \\
\hline Lesotho & 4 & 4 \\
\hline Liberia & 25 & 3 \\
\hline Libyen & 25 & 25 \\
\hline Luxemburg & 25 & 25 \\
\hline Madagaskar & 25 & 25 \\
\hline Malawi & 25 & 25 \\
\hline Malaysia & 25 & 25 \\
\hline Mali & 25 & 8 \\
\hline Marokko & 25 & 25 \\
\hline Mauretanien & 25 & 20 \\
\hline Mauritius & 16 & 24 \\
\hline Mexiko & 25 & 13 \\
\hline Mozambique & 19 & 19 \\
\hline Myanmar & 22 & 22 \\
\hline Namibia & 7 & 7 \\
\hline Nepal & 25 & 12 \\
\hline Neusecland & 25 & 25 \\
\hline Nicaragua & 19 & 17 \\
\hline Niederlande & 25 & 25 \\
\hline Niger & 25 & 25 \\
\hline Nigeria & 25 & 25 \\
\hline Norwegen & 25 & 25 \\
\hline Oman & 25 & 25 \\
\hline Osterreich & 25 & 25 \\
\hline Pakistan & 25 & 25 \\
\hline Panama & 25 & 4 \\
\hline Papua Neu Guinea & 25 & 25 \\
\hline Paraguay & 25 & 4 \\
\hline Peru & 25 & 16 \\
\hline Philippinen & 25 & 25 \\
\hline Polen & 0 & 3 \\
\hline Portugal & 25 & 25 \\
\hline Republik Kongo & 14 & 18 \\
\hline
\end{tabular}




\begin{tabular}{|c|c|c|}
\hline Land & N Handelsregulierung & N Kapitalverkehrsregulierung \\
\hline Ruanda & 25 & 8 \\
\hline Sambia & 25 & 16 \\
\hline Saudi Arabien & 25 & 5 \\
\hline Schweden & 25 & 25 \\
\hline Schweiz & 25 & 7 \\
\hline Senegal & 16 & 25 \\
\hline Sierra Leone & 19 & 22 \\
\hline Simbabwe & 23 & 23 \\
\hline Slowakische Republik & 0 & 2 \\
\hline Somalia & 17 & 17 \\
\hline Spanien & 25 & 25 \\
\hline Sri lanka & 25 & 25 \\
\hline Sudafrika & 7 & 25 \\
\hline Sudan & 25 & 25 \\
\hline Südkorea & 25 & 25 \\
\hline Syrien & 25 & 25 \\
\hline Tansania & 25 & 25 \\
\hline Thailand & 25 & 25 \\
\hline Togo & 25 & 25 \\
\hline Trinidad und Tobago & 25 & 25 \\
\hline Tschad & 25 & 25 \\
\hline Tschechische Republik & 0 & 3 \\
\hline Tunesien & 25 & 25 \\
\hline Türkei & 25 & 25 \\
\hline Uganda & 25 & 25 \\
\hline Ungarn & 0 & 3 \\
\hline Uruguay & 8 & 8 \\
\hline V. R. China & 23 & 23 \\
\hline Venezuela & 25 & 4 \\
\hline Vereinigte Staaten von Amerika & 25 & 13 \\
\hline Vietram & 25 & 21 \\
\hline Zentralafrikanische Republik & 25 & 24 \\
\hline
\end{tabular}

\section{Literatur}

Anselin, Luc, 1988: Spatial Econometrics: Methods and Models. Boston.

Arteta, Carlos/Eichengreen, Barry/Wyplosz, Charles, 2001: When Does Capital Account Liberalization Help More than it Hurts?, in: National Bureau of Economic Research Working Paper, 8414, Cambridge.

Balassa, Bela, 1965: Tariff Protection in Industrial Countries: An Evaluation, in: Journal of Political Economy 73, 573-594.

Barro, Robert J./Lee, Jong-Wha, 1993: International Comparison of Educational Attainment, in: Journal of Monetary Economics 32, 363-394.

Beck, Nathaniel/Katz, Jonathan N., 1995: What to Do (And Not to Do) With Time-Series CrossSection Data, in: American Political Science Review, 89, 634-647.

Bhagwati, Jagdish N., 1965: On the Equivalence of Tariffs and Quotas, in: Baldwin, Robert E. (Hrsg.), Trade, Growth and the Balance of Payments. Chicago, 53-67.

Bodenstein, Thilo, 2005: Wege zum Weltmarkt. Wiesbaden.

Bodenstein, Thilo/Schneider, Gerald, 2006: Capitalist Junctures: Explaining Economic Openness in the Transition Countries, in: European Journal of Political Research 45, 467-497. 
Bruno, Michael/Easterly, William, 1996: Tales of Crisis that Beget Reforms, in: National Bureau of Economic Research Working Paper, 5452. Cambridge.

Bussmann, Margit/Schneider, Gerald, 2007: When Globalization Discontent Turns Violent: Foreign Economic Liberalization and Internal War, in: International Studies Quarterly 51, 79-97.

Bussmann, Margit/Schneider, Gerald/Wiesehomeier, Nina, 2005: Foreign Economic Liberalization and Civil War in Sub-Saharan Africa, in: European Journal of International Relations 11, 551-579.

Bussmann, Margit/Schneider, Gerald/Wiesehomeier, Nina, 2006: Globalization Anger? Foreign Economic Liberalization and Social Unrest in Latin America and the Caribbean. Unveröffentlichtes Manuskript.

Corden, Warner Max, 1966: The Structure of a Tariff System and the Effective Protection Rate, in: Journal of Political Economy 74, 221-247.

Downs, Anthony, 1957: An Economic Theory of Democracy. New York.

Drazen, Allan/Grilli, Vittorio, 1993: The Benefit of Crisis for Economic Reforms, in: American Economic Review 83, 598-607.

Drezner, Daniel W., 2005: Globalization, Harmonization, and Competition: The Different Pathways to Policy Convergence, in: Journal of European Public Policy 12, 841-859.

Durlauf, Steven N./Johnson, Paul A., 1995: Multiple Regimes and Cross-Country Growth Behavior, in: Journal of Applied Econometrics 10, 365-384.

Edwards, Sebastian, 2001: Capital Mobility and Economic Performance: Are Emerging Economies Different?, in: National Bureau of Economic Research Working Paper Series 8076. Cambridge.

Elkins, Zachary/Guzman, Andrew/Simmons, Beth, 2006: Competing for Capital: The Diffusion of Bilateral Investment Treaties, 1960-2000, in: International Organization 60, 811-846.

Feldstein, Martin/Horioka, Charles, 1980: Domestic Saving and International Capital Flows, in: Economic Journal 90, 314-329.

Fernández, Raquel, 1997: Returns to Regionalism: An Evaluation of Non-Traditional Gains from RTAs, in: National Bureau of Economic Research Working Paper \#5970. Cambridge.

French, Kenneth A./Poterba, James M., 1991: Investor Diversification and International Equity Markets, in: American Economic Review 81, 222-226.

Friedman, Thomas L., 2005: The World is Flat. A Brief History of the Twenty-First Century. New York.

Galor, Oded, 1996: Convergence? Inference from Theoretical Models, in: Economic Journal 106, 1056-1069.

Garrett, Geoffrey, 1998a: Global Markets and National Politics: Collision Course or Virtuous Circle?, in: International Organization 52, 787-824.

Garrett, Geoffrey, 1998b: Partisan Politics in the Global Economy. Cambridge.

Garrett, Geoffrey, 2000: The Causes of Globalization, in: Comparative Political Studies 33, 941 991.

Green, Donald P./Kim Soo Yeon/Yoon, David H., 2001: Dirty Pool, in: International Organization $55,441-468$.

Grossman, Gene M./Helpman, Elhanan, 1995: The Politics of Free Trade Agreements, in: American Economic Review 84, 667-690.

Grossman, Gene/Helpman, Elhanan, 1994: Protection for Sale, in: American Economic Review 84, 833-850.

Harberger; Arnold C., 1993: The Search for Relevance in Economics, in: American Economic Review Papers and Proceedings 83, 1-17.

Harrison, Ann/Hanson, Gordon, 1999: Who Gains From Trade Reform? Some Remaining Puzzles, in: Journal of Development Economics 59, 125-154.

Irwin, Douglas A., 1996: Against the Tide. An Intellectual History of Free Trade. Princeton.

Johnson, Harry, 1965: The Theory of Tariff Structure with Special Reference to World Trade and Development, in: Johnson, Harry/Kenen, Peter(Hrsg.), Trade and Development. Geneva, 9-29.

Kornai, Janos, 1992: The Socialist System: The Political Economy of Communism. Princeton. 
Krishna, Pravin/Panagariya, Arvind, 2000: A Unification of Second Best Results in International Trade, in: Journal of International Economics 52, 235-257.

Leamer, Edward E., 1988: Measures of Openness, in: Baldwin, Robert E. (Hrsg.), Trade Policy Issues and Empirical Analysis. Chicago, 147-200.

Lee, Jong-Wha, 1993: International Trade, Distortions and Long-Run Economic Growth, in: International Monetary Fund Staff Papers 40, Washington, 299-328.

Martin, Christian W., 2004: Demokratie, Autokratie und die regulative Gestaltung der Außenwirtschaftsbeziehungen in Entwicklungsländern, in: Politische Vierteljahresschrift 46, 32-54.

Martin, Christian W., 2005: Die doppelte Transformation. Demokratie und Außenwirtschaftsliberalisierung in Entwicklungsländern. Wiesbaden.

Mukand, Sharun W./Rodrik, Dani, 2005: In Search of the Holy Grail: Policy Convergence, Experimentation, and Economic Performance, in: American Economic Review 95 (1), 374-383.

Oneal, John R./Russett, Bruce, 2001: Clear and Clean: The Fixed Effects of the Liberal Peace, in: International Organization 55, 469-485.

Quinn, Dennis, 1997: The Correlates of Change in the International Financial Regulation, in: American Political Science Review 91, 531-551.

Ritzer, George, 1993: The McDonaldization of Society. Thousand Oaks.

Rodrik, Dani, 1992a: The Limits of Trade Policy Reform in Developing Countries, in: Journal of Economic Perspectives 6, 87-105.

Rodrik, Dani, 1992b: The Rush to Free Trade in the Developing World: Why So Late? Why Now? Will it Last? in: National Bureau of Economic Research Working Paper, 3947. Cambridge.

Rodrik, Dani, 2001: Trading in Illusions, in: Foreign Policy 123, 55-62.

Rosser, J. Barcley, 1999: On the Complexities of Complex Economic Dynamics, in: Journal of Economic Perspectives 13, 169-192.

Sachs, Jeffrey/Warner, Andrew, 1995: Economic Reform and the Process of Global Integration, in: Brookings Papers on Economic Activity 1, 1-118.

Sinn, Hans-Werner, 1990: Tax Harmonization and Tax Competition in Europe, in: European Economic Review 34, 489-504.

Simmons, Beth A./Elkins, Zachary, 2004: The Globalization of Liberalization: Policy Diffusion in the International Political Economy, in: American Political Science Review 98, 171-190.

Stiglitz, Joseph, 2002: Globalization and its Discontents. New York.

Svensson, Jakab, 1998: Investment, Property Rights and Political Instability: Theory and Evidence, in: European Economic Review 42, 1317-1341.

Torrens, Robert, 1821: An Essay on the Production of Wealth. London.

Torrens, Robert, 1833: Letters on Commercial Policy. London.

Torrens, Robert, 1844: The Budget: On Commercial and Colonial Policy. London.

Viner, Jacob, 1950: The Customs Union Issue. New York.

World Bank, 2001: World Development Indicators 2000. Washington, D.C.

World Bank, 1987: World Development Report. Washington, D.C.

World Bank, 1994: Adjustment in Africa: Reforms, Results, and the Road Ahead. Washington, D.C. 